\title{
HLLC solver for ideal relativistic MHD
}

\author{
V. Honkkila, P. Janhunen* \\ University of Helsinki, Department of Physical Sciences, P.O.Box 64 FIN- \\ 00014, Helsinki, Finland \\ *Also at Finnish Meteorological Institute, Space Research, P.O.Box 503, FIN- \\ 00101, Helsinki, Finland \\ email: Ville.Honkkila@helsinki.fi, Pekka.Janhunen@fmi.fi
}

\begin{abstract}
An approximate Riemann solver of Godunov type for ideal relativistic magnetohydrodynamic equations (RMHD) named as HLLC ("C" denotes contact) is developed. In HLLC the Riemann fan is approximated by two intermediate states, which are separated by the entropy wave. Numerical tests show that HLLC resolves contact discontinuity more accurately than the Harten-Lax-van Leer (HLL) method and an isolated contact discontinuity exactly.
\end{abstract}

\section{Introduction}

Since the complexity of the special relativistic ideal magnetohydrodynamics (RMHD), numerical simulations are usually the only means to study the rich structure of their solutions. The RMHD has been made use of in many astrophysical phenomena, for instance those involving relativistic jets, which have been recently started to study by numerical simulations [12].

There are several requirements for the numerical simulation in general such as accuracy, robustness and computational efficiency and several different approaches has been developed in order to fulfill them in the most succesful way. The approximate Riemann solvers of Godunov type have proven to be applicable in MHD and RMHD simulations. Among them is the HLL solver and the HLLC solver $[7,6,3,11]$. The latter has recently been applied succesfully to RHD and RMHD even including higher order multidimensional cases [13, 14].

Our goal is to find a conservative and positive solver with minimum diffusion which would still be fast enough. Positivity means that the solver maintains the condition $\rho \geq 0, p \geq 0$. One way to achieve these goals, which is followed here, is to add gradually intermediate states to HLL which has one intermediate state [15]. In this paper a HLLC solver containing two intermediate 
states is introduced for RMHD equations and it is shown by numerical examples that it resolves contact wave more accurately than the HLL method. The scheme presented here differs from [14] and the differences will be discussed in the Summary.

\section{Theory}

\section{$2.1 \quad$ RMHD equations}

The ideal special relativistic magnetohydrodynamical equations (RMHD) [1, $5,8]$ are

the conservation of mass

$$
\frac{\partial}{\partial x^{\alpha}}\left(\rho u^{\alpha}\right)=0
$$

the conservation of energy-momentum

$$
\frac{\partial}{\partial x^{\alpha}}\left(T_{F L}^{\alpha \beta}+T_{E M}^{\alpha \beta}\right)=0
$$

the Maxwell equations

$$
\frac{\partial}{\partial x^{\alpha}}\left(u^{\alpha} b^{\beta}-b^{\alpha} u^{\beta}\right)=0
$$

together with the state equation in the form $e=e(p, \rho)$. We are using

$$
\text { the } \gamma \text { - law equation of state }
$$

$$
e=\rho c^{2}+p /(\gamma-1)
$$

Greek indices range over $0,1,2,3$ and Latin indices over 1,2,3, where 0 is indicating the time component and 1,2,3 the space components. In the equations $x^{\alpha}=\left(c t, x^{j}\right)$ is the four vector of space-time coordinates, $\Gamma=\left(1-v^{2} / c^{2}\right)^{-1 / 2}$ is the Lorentz factor, $u^{\alpha}=\left(\Gamma c, \Gamma v^{j}\right)$ is the four velocity, $\gamma$ is the polytropic (adiabatic) index and

$$
b^{\alpha}=\left(\Gamma(\vec{v} / c \cdot \vec{B}), B^{j} / \Gamma+\Gamma(\vec{v} / c \cdot \vec{B}) v^{j} / c\right)
$$

is the magnetic induction four vector. The energy-momentum tensor for relativistic ideal fluid is

$$
T_{F L}^{\alpha \beta}=(e+p) u^{\alpha} u^{\beta} / c^{2}+p g^{\alpha \beta}
$$

where $g^{\alpha \beta}=\operatorname{diag}(-1,1,1,1)$ is the Minkowski metric tensor. The electromagnetic energy-momentum tensor is

$$
T_{E M}^{\alpha \beta}=\epsilon_{0} F_{\gamma}^{\alpha} F^{\beta \gamma}-\frac{\epsilon_{0}}{4} g^{\alpha \beta} F_{\gamma \delta} F^{\gamma \delta},
$$


where $F^{\alpha \beta}$ is the electromagnetic field tensor, which in the ideal infinite conductivity approximation $\overrightarrow{\mathcal{E}}=\vec{B} \times \vec{v}$ becomes

$$
F^{\alpha \beta}=\epsilon^{\gamma \delta \alpha \beta} b_{\gamma} u_{\beta}
$$

where $\epsilon$ is the Levi-Civita permutation symbol.

RMHD equations written out explicitly in the usual $1+3$ conservative form are

$$
\begin{gathered}
\frac{\partial U}{\partial t}+\sum_{i=1}^{3} \frac{\partial F_{i}(U)}{\partial x_{i}}=0, \\
U \equiv\left(\begin{array}{c}
D \\
\vec{k} \\
E \\
\vec{B}
\end{array}\right)=\left(\begin{array}{c}
\Gamma \rho \\
\Gamma^{2}(e+p) \vec{v} / c^{2}+\vec{S} / c^{2} \\
\Gamma^{2}(e+p)-p+e^{A} \\
\vec{B}
\end{array}\right), \\
F_{i}=\left(\begin{array}{c}
\Gamma \rho v_{i} \\
\frac{\Gamma^{2}}{c^{2}}(e+p) v_{i} \vec{v}+p \sum_{j=1}^{3} \delta_{i j} \vec{e}_{j}+\vec{P}_{i}^{A} \\
\Gamma^{2}(e+p) v_{i}+\vec{S} \\
v_{i} \vec{B}-B_{i} \vec{v},
\end{array}\right)
\end{gathered}
$$

where

$$
\begin{aligned}
\vec{S} & =\frac{1}{\mu_{0}}(\overrightarrow{\mathcal{E}} \times \vec{B}) \\
\overrightarrow{\mathcal{E}} & =\vec{B} \times \vec{v} \\
e^{A} & =\frac{1}{2 \mu_{0}}\left(B^{2}+\frac{1}{c^{2}} \mathcal{E}^{2}\right) \\
\vec{P}_{i}^{A} & =e^{A} \sum_{j=1}^{3} \delta_{i j} \vec{e}_{j}-\frac{1}{\mu_{0}} B_{i} \vec{B}-\frac{1}{\mu_{0} c^{2}} \mathcal{E}_{i} \overrightarrow{\mathcal{E}}
\end{aligned}
$$

Here $U=(D, \vec{k}, E, \vec{B})$ is the 8-vector of conserved variables mass-, momentumand energy density and magnetic field and $F_{i}$ is the 8-vector of corresponding fluxes. We introduce also the vector of primitive variables $W=(\rho, \vec{v}, p, \vec{B})$ where $\rho$ is mass density, $\vec{v}$ velocity and $p$ pressure. The numerical discretization below uses the RMHD equations in the form (9).

\subsection{Numerical method}

RMHD equations like MHD have seven eigenvalues which correspond to entropy wave, two Alfvén waves and four (slow/fast) magnetosonic waves. A solution of the Riemann problem may include shock-, rarefaction-, compoundand overcompressible shock waves. Let us consider the one-dimensional case 
from now on $\left(F \equiv F_{x}\right)$. The numerical solution of the Riemann problem can be written in conservative form

$$
U_{i}^{n+1}=U_{i}^{n}-\frac{\Delta t}{\Delta x}\left(F_{n u m}\left(U_{i}^{n}, U_{i+1}^{n}\right)-F_{\text {num }}\left(U_{i-1}^{n}, U_{i}^{n}\right)\right),
$$

where $F_{\text {num }}$ is the numerical flux function, $n$ refer to time step and $i$ to cell number. Note that the flux $F_{i}$ is given in (11) as a function of primitive variables: $U=m(W)$ where $m$ is the mapping defined by (10). That means that the inverse map $U \mapsto m^{-1}(U)=W$ should be determined, because the time stepping process (16) produces next time level for the conservative variables not the primitive ones. The detailed description of the inverse mapping is given in [5]. Here we repeat some of the key points. The primitive variables can be written

$$
\begin{aligned}
& \rho=D \sqrt{1-v^{2} / c^{2}}, p=\left(\left(1-v^{2} / c^{2}\right) H c^{2}-\rho c^{2}\right) / \gamma_{1}, \\
& \vec{v}=\frac{1}{H+\epsilon_{0} B^{2}}\left(\vec{k}+(\vec{k} \cdot \vec{B}) \epsilon_{0} \vec{B} / H\right),
\end{aligned}
$$

where $H=\Gamma^{2}\left(\rho+\gamma_{1} p / c^{2}\right)$ and $\gamma_{1}=\gamma /(\gamma-1)$. In (17) the primitive variables are functions of $U, v^{2}$ and $H$ and thus we require two more equations to express $v^{2}$ and $H$ in terms of $U$. These are obtained from the energy and momentum density definitions (10). The momentum squared is

$$
H^{2} v^{2}+\left(2 H+\epsilon_{0} B^{2}\right)\left(\epsilon_{0} B^{2} v^{2}-(\vec{k} \cdot \vec{B})^{2} / H^{2}\right) /\left(H+\epsilon_{0} B^{2}\right)^{2}-k^{2}=0
$$

and the equation for the energy density can be put into the form

$$
\begin{aligned}
& \left(\left(1-\frac{1-v^{2} / c^{2}}{\gamma_{1}}\right) H c^{2}-E+D c^{2} \sqrt{1-v^{2} / c^{2}} / \gamma_{1}+\frac{B^{2}}{\mu_{0}}\right)\left(H+\epsilon B^{2}\right)^{2}+ \\
& \epsilon_{0}\left(B^{2} k^{2}-(\vec{k} \cdot B)^{2}\right) / 2=0
\end{aligned}
$$

The equation (19) is a third order polynomial equation for $H$ so it can be solved analytically. It appears that all the roots are real and the correct one is the largest one. The root is substituted in (18) and we get equations of the form

$$
\mathcal{F}(\zeta)=0, \quad \frac{d \mathcal{F}(\zeta)}{d \zeta}=0, \quad \zeta \equiv v^{2}
$$

from which $v^{2}$ is solved numerically by Newton's method. Therefore we know both $v^{2}$ and $H$ as a function of conservative variables and the primitive variables as functions of the conservative variables can be solved from (17). Thus the inverse mapping $U \mapsto m^{-1}(U)=W$ has been determined.

In a two-state approximate Riemann solver (HLLC) $[7,6]$ the Riemann fan is approximated by two intermediate states $U_{L, R}^{*}$, which are separated by the 
line $d x / d t=S_{M}$, where $S_{M}$ is the eigenvalue of the entropy wave in RMHD. The numerical flux function becomes

$$
F_{\text {num }} \equiv F_{H L L C}=\left\{\begin{array}{cc}
F_{L}, & S_{L}>0, \\
F_{L}^{*}, & S_{L} \leq 0 \leq S_{M}, \\
F_{R}^{*}, & S_{M} \leq 0 \leq S_{R}, \\
F_{R} & S_{R}<0,
\end{array}\right.
$$

where $S_{L, R}$ are the minimum and maximum signal speeds in the system, which in ideal RMHD are the speeds of magnetosonic waves $\lambda_{1,7}\left(\lambda_{1}<\lambda_{7}\right)$. They can be solved analytically from the quartic equation

$$
\left(1-\epsilon^{2}\right)\left(u^{0} \lambda-u^{x}\right)^{4}+\left(1-\lambda^{2}\right)\left(c_{s}^{2}\left(\tilde{b}^{0} \lambda-\tilde{b}^{x}\right)^{2}-\epsilon^{2}\left(u^{0} \lambda-u^{x}\right)^{2}\right)=0,
$$

where $c_{s}=(\gamma p / w)^{1 / 2}$ is the sound speed, $\tilde{b}^{\alpha}=b^{\alpha} /\left(w_{t o t}\right)^{1 / 2}, w_{t o t}=w+b_{\alpha} b^{\alpha}$ and $w=e+p$ is the enthalpy. After $\lambda_{1,7}$ are solved from (22) the speeds $S_{L, R}$ are calculated in the numerical code as in [4]

$$
S_{L}=\min \left(\lambda_{1}\left(U_{L}\right), \lambda_{1}\left(U_{R}\right)\right), \quad S_{R}=\max \left(\lambda_{7}\left(U_{L}\right), \lambda_{7}\left(U_{R}\right)\right)
$$

Another possibility is to define $S_{L}=-c, S_{R}=c$, but this adds diffusion to the solution.

Now we need to define the fluxes $F_{L, R}^{*}$. The integral form of the conservative equations (9) is

$\int_{x_{1}}^{x_{2}} U\left(x, t_{2}\right) d x-\int_{x_{1}}^{x_{2}} U\left(x, t_{1}\right) d x+\int_{t_{1}}^{t_{2}} F\left(U\left(x_{2}, t\right)\right) d t-\int_{t_{1}}^{t_{2}} F\left(U\left(x_{1}, t\right)\right) d t=0$.

By choosing different values for $\left(x_{1,2}, t_{1,2}\right)$ the Rankine-Hugoniot jump conditions across the $S_{L}, S_{M}$ and $S_{R}$ waves are derived

$$
\begin{aligned}
& S_{\alpha}\left(U_{\alpha}^{*}-U_{\alpha}\right)=F_{\alpha}^{*}-F_{\alpha}, \quad \alpha=L, R, \\
& S_{M}\left(U_{R}^{*}-U_{L}^{*}\right)=F_{R}^{*}-F_{L}^{*} .
\end{aligned}
$$

It would seem natural to define $F_{\alpha}^{*} \equiv F\left(U_{\alpha}^{*}\right)$, but then the flux would not be consistent with the conservation law over the rectangle $\left(i-\frac{1}{2}, i\right) \times(0, \Delta t)[3]$.

$$
\text { i) } B_{x} \neq 0
$$

We use the knowledge that the jump condition over the entropy wave $S_{M}$ (26) leads to the following relations when $B_{x} \neq 0[1,8]$

$$
\rho_{L}^{*} \not \equiv \rho_{R}^{*}, \quad v_{i L}^{*}=v_{i R}^{*}, \quad p_{L}^{*}=p_{R}^{*}, \quad B_{i L}^{*}=B_{i R}^{*}, \quad i=x, y, z .
$$

Let us construct a two-state solver [6] so that the intermediate states partly correspond to the single-state HLL fluxes in order to satisfy (27). First we 
define $S_{M}=v_{x \alpha}^{*}=v_{x}^{*}(\alpha=L, R)$ as it was also done in $[16,3,15,6]$ and furthermore

$$
\begin{aligned}
& v_{i \alpha}^{*}=v_{i}^{*}, \\
& p_{\alpha}^{*}=p^{*}, \\
& B_{i \alpha}^{*}=B_{i}^{*}, i=x, y, z .
\end{aligned}
$$

From $(26),(28)$ it follows

$$
\rho_{\alpha}^{*}=\frac{\Gamma \rho_{\alpha}\left(S_{\alpha}-v_{x \alpha}\right)}{\Gamma^{*}\left(S_{\alpha}-S_{M}\right)}, \quad \Gamma^{*} \equiv\left(1-v^{* 2} / c^{2}\right)^{-1 / 2} .
$$

The primitive 1-state HLL variables $p^{*}, v_{i}^{*}, B_{i}^{*}$ are obtained from

$$
W^{*}=m^{-1}\left(U^{*}\right),
$$

where

$$
U^{*}=\frac{S_{R} U_{R}-S_{L} U_{L}-F_{R}+F_{L}}{S_{R}-S_{L}}
$$

are HLL averages. Now the intermediate two-state primitive variables $W_{\alpha}^{*}$ are defined according to equations (28-33) and further intermediate two-state conservative variables by $U_{\alpha}^{*}=m\left(W_{\alpha}^{*}\right)$. Thus the intermediate two state fluxes $F_{\alpha}^{*}$ can be calculated from (25), (28-33) and the time step from $(16,21)$. The mapping $m^{-1}$ is also needed in calculating the values for the fluxes $F_{L, R}$, because they are given as functions of the primitive variables $W$ in (11) and the time-stepping is done for conservative variables $U$. Formally this can be written as $F(W)_{L, R}=F\left(m^{-1}(U)\right)_{L, R}$.

ii) $B_{x}=0$

In the case of $B_{x}=0$ the jump conditions over the $S_{M}$ wave [1,9] yield

$$
v_{x \alpha}^{*}=v_{x}^{*}, p_{L}^{*}+\frac{b_{L}^{* 2}}{2 \mu_{0}}=p_{R}^{*}+\frac{b_{R}^{* 2}}{2 \mu_{0}}, \alpha=L, R
$$

where $b_{\alpha}^{* 2}=B_{\alpha}^{* 2}\left(1-\left(\frac{v_{\alpha}^{*}}{c}\right)^{2}\right)+\left(\frac{\vec{v}_{\alpha}^{*}}{c} \cdot \vec{B}_{\alpha}^{*}\right)^{2}$. Now we assume identically to the $B_{x} \neq 0$ case that $S_{M}=v_{x \alpha}^{*}=v_{x}^{*}$ and similarly

$$
p_{\alpha}^{*}+\frac{b_{\alpha}^{* 2}}{2 \mu_{0}}=p^{*}+\frac{b^{* 2}}{2 \mu_{0}} \equiv p_{t o t}^{*},
$$

where $p^{*}, b^{*}$ are obtained from the HLL average states. From the jump condi- 
tions over the $S_{\alpha}$ waves (25) it follows that

$$
\begin{aligned}
& v_{y, z \alpha}^{*}=\left(C_{y, z \alpha}\left(S_{\alpha} k_{y, z \alpha}-F_{\alpha}^{k_{y, z}}\right)-C_{\alpha}\left(S_{\alpha} k_{z, y \alpha}-F_{\alpha}^{k_{z, y}}\right)\right) /\left(C_{y} C_{z}-C_{\alpha}^{2}\right), \\
& \rho_{\alpha}^{*}=\frac{\Gamma_{\alpha} \rho_{\alpha}}{\Gamma_{\alpha}^{*}} \frac{\left(S_{\alpha}-v_{x \alpha}\right)}{\left(S_{\alpha}-S_{M}\right)}, E_{\alpha}^{*}=\frac{S_{\alpha} E_{\alpha}-k_{x \alpha}+p_{t o t}^{*} S_{M}}{S_{\alpha}-S_{M}}, \\
& B_{y, z \alpha}^{*}=B_{y, z \alpha} \frac{\left(S_{\alpha}-v_{x \alpha}\right)}{\left(S_{\alpha}-S_{M}\right)}
\end{aligned}
$$

where

$$
\begin{aligned}
& C_{\alpha}=\left(S_{M}-S_{\alpha}\right) \frac{B_{y \alpha}^{*} B_{z \alpha}^{*}}{\mu_{0} c^{*}}, C_{y, z \alpha}=\left(S_{\alpha}-S_{M}\right)\left(G_{\alpha}+\frac{B_{y, z \alpha}^{* 2}}{\mu_{0} c^{2}}\right), \\
& G_{\alpha}=\left(S_{\alpha} E_{\alpha}-F_{\alpha}^{E}+S_{\alpha} p_{t o t}^{*}\right) /\left(c^{2}\left(S_{\alpha}-S_{M}\right)\right)-\frac{B_{\alpha}^{* 2}}{\mu_{0} c^{2}}
\end{aligned}
$$

where $F_{\alpha}^{E}, F_{\alpha}^{k_{y, z}}$ refer to the energy and momentum parts of the flux function (11) respectively. The momentum densities of the intermediate states are obtained from the definitions $k_{i \alpha}^{*}=\left(E_{\alpha}^{*}+p_{t o t}^{*}\right) v_{i}^{*}-\frac{1}{\mu_{0} c^{*}} B_{i}^{*} \vec{v}^{*} \cdot \vec{B}^{*}$. The rest of the implementation details follow the $B_{x} \neq 0$ case.

\section{Numerical tests}

A few numerical test problems for RMHD are described in [2, 5]. Three of them are performed here with the same numerical and physical parameters. The number of gridpoints is 1600 and the final time is 0.4 . The polytropic index is $\gamma=5 / 3$, the constants $\mu_{0}, \epsilon_{0}$ are normalized to unity and therefore also $c$. We present here results for the tests 1,2 and 4 in $[2,5]$ (here they are numbered 1,2,3) and three other tests. Tests are performed for both 1-state HLL and 2-state HLLC solvers.

Test 0a (Fig.1) is defined by the initial state at time $t=0$ as

$$
W=\left(\rho, v_{x}, v_{y}, v_{z}, p, B_{x}, B_{y}, B_{z}\right)= \begin{cases}(1,0,0.4,0,1,1,1,0) & \text { for } x<x_{h} \\ (0.125,0,0.4,0,1,1,1,0) & \text { for } x>x_{h}\end{cases}
$$

where $x_{h}$ is the middle point of the grid.

Test 0b (Fig.2):

$$
W(t=0)= \begin{cases}(1,0.2,0,0,1,1,1,0) & \text { for } x<x_{h} \\ (0.125,0.2,0,0,1,1,1,0) & \text { for } x>x_{h}\end{cases}
$$

These two tests represent an isolated contact discontinuity where there is a jump only in rest mass density $\rho$. Only the rest mass density $\rho$ profiles are plotted because the other primitive variables are constant. The results show that HLLC resolves an isolated discontinuity exactly when $v_{x}=0$ i.e. in a coordinate system where the contact discontinuity is at rest. The difference between HLL and HLLC becomes smaller when $v_{x}$ approaches to $c=1$. 
Test 1 (Fig.3,4):

$$
W(t=0)= \begin{cases}(1,0,0,0,1,0.5,1,0) & \text { for } x<x_{h} \\ (0.125,0,0,0,0.1,0.5,-1,0) & \text { for } x>x_{h}\end{cases}
$$

This test shows clearly that the contact discontinuity, located approximately at $x=0.6$, is resolved more accurately by the HLLC than the HLL solver.

Test 2 (Fig.5,6):

$$
W(t=0)= \begin{cases}(1,0,0,0,30,5,6,6) & \text { for } x<x_{h} \\ (1,0,0,0,1,5,0.7,0.7) & \text { for } x>x_{h}\end{cases}
$$

In this test there is a contact discontinuity approximately at $x=0.78$. The HLLC solver resolves it more accurately than the HLL solver, but the difference is small.

Test 3 (Fig. 7,8$)$ :

$$
W(t=0)= \begin{cases}(1,0.999,0,0,0.1,10,7,7) & \text { for } x<x_{h} \\ (1,-0.999,0,0,0.1,10,-7,-7) & \text { for } x>x_{h}\end{cases}
$$

Two ultra relativistic streams collide producing a lower dip in the rest mass density profile at $x=0.5$. The HLLC solver captures this feature better than the HLL solver.

The tests 1,2,3 show that HLLC method restores the contact discontinuity in the mass density calculations. The difference between HLL and HLLC in resolving the other primitive variables is neglible.

Test $\mathbf{1 b}$ (Fig.9,10):

$$
W(t=0)= \begin{cases}(1,0,0,0,1,0,1,0) & \text { for } x<x_{h} \\ (0.125,0,0,0,0.1,0,-1,0) & \text { for } x>x_{h}\end{cases}
$$

From the results of the test $1 \mathrm{~b}$ it can be seen that in the $B_{x}=0$ case the HLLC solver resolves the contact discontinuity, located at $x=0.58$, more sharply than the HLL solver in mass density, pressure and magnetic field $\left(v_{y, z}\right.$ are constants in this test).

\section{Summary and conclusions}

In this paper we have started succesfully our program of developing a robust (positive) solver for RMHD equations by gradually adding intermediate states to HLL [15]: a solver for RMHD with two intermediate states, HLLC, has been developed. It has been shown by numerical tests that the HLLC has the benefit of realizing the contact discontinuity more accurately than HLL and in particular HLLC resolves an isolated contact discontinuity exactly. In several tests positivity has always been maintained. Numerical tests show that 
the scheme corresponds to the HLLC for MHD in the limit $v / c \rightarrow 0$ provided that the variable range is such that the magnetosonic wave speeds according to MHD are below the speed of light. This is the case when the classical sound speed and Alfvén speed are below the speed of light.

There are some essential differences between our scheme and the HLLC scheme developed in [14]. The speed of the entropy wave $S_{M} \equiv v_{x}^{*}$ and the pressure of the intermediate state in the $B_{x} \neq 0 p^{*}$ are defined differently. We have $S_{M}=v_{x}\left(U^{*}\right)$ and $p^{*}=p\left(U^{*}\right)$. In [14] $S_{M}, p^{*}$ are considered as auxiliary variables and they are solved from the consistency conditions. Also the definitions of the transverse velocity components $v_{y}^{*}, v_{z}^{*}$ are different. Especially we have not found any problems that $v_{y}^{*}, v_{z}^{*}$ would become ill-defined when $B_{x} \rightarrow 0$ which was observed by [14] in their scheme. In the scheme by [14] there is a problem with the definitions of the intermediate transverse velocities $v_{y}^{*}, v_{z}^{*}$, which can become arbitrarily large when $B_{x} \rightarrow 0$. This leads in certain circumstances to the situation where the positivity is lost. We have not encountered those problems and $v_{y}^{*}, v_{z}^{*}$ remain well defined by construction because they are formed from the HLL averages.

The HLLC solver for RMHD could be applied to astrophysical phenomena such as relativistic jets. In future work we intend to increase intermediate states in order to improve the accuracy of resolving the other existing discontinuities.

\section{References}

[1] A.M. Anile, Relativistic fluids and magneto-fluids, (Cambridge University Press, Cambridge), (1989).

[2] D.S. Balsara, Total variation diminishing scheme for relativistic magnetohydrodynamics, ApJS, 132, p. 83, (2001).

[3] P. Batten, N.Clarke, C. Lambert, D.M. Causon, On the choice of wave speeds for the HLLC Riemann solver, SIAM J. Sci. Stat. Comp., 35, p. 1553, (1997).

[4] S.F.Davis, Simplified second-order Godunov-type methods, SIAM J. Sci. Statist. Comput., 9, p. 445, (1988).

[5] L. Del Zanna, N. Bucciantini, An efficient shock-capturing central-type scheme for multidimensional relativistic flows, $A \& \mathcal{E} A$, 390, p. 1177, (2002).

[6] K.F. Gurski, An HLLC-type approximate Riemann solver for ideal magnetohydrodynamics, SIAM J.Sci. Comp., 25, p. 2165, (2004). 
[7] A. Harten, P.D. Lax, B. van Leer, On upstream differencing and Godunov-type schemes for hyperbolic conservation laws, SIAM Rev., 25, p. 35, (1983).

[8] A.V. Koldoba, O.A. Kuznetsov, G.V. Ustyugova, An approximate Riemann solver for relativistic magnetohydrodynamics, Mon. Not. R. Astron. Soc., 333, p. 932, (2002).

[9] A. Lichnerowicz, Relativistic Hydrodynamics and Magnetohydrodynamics, (Benjamin Press, New York), (1967).

[10] R.J. LeVeque, Nonlinear Conservation Laws and Finite Volume Methods for Astrophysical Fluid Flow, in Computational Methods for Astrophysical Fluid Flow, Springer Verlag, 1998.

[11] S. Li, An HLLC Riemann solver for magneto-hydrodynamics, J. Comp. Phys., 203, p. 344, (2005).

[12] D.L. Meier, S. Koide, Y. Uchida, Magnetohydrodynamic production of relativistic jets, Science, 291, p. 84, (2001).

[13] A. Mignone, G. Bodo, An HLLC solver for relativistic flows-I. Hydrodynamics, Mon. Not. R. Astron. Soc., 364, p. 126, (2005).

[14] A. Mignone, G. Bodo, An HLLC solver for relativistic flows-II. Magnetohydrodynamics, Mon. Not. R. Astron. Soc., 368, p. 1040, (2006).

[15] T. Miyoshi, K. Kusano, A multi-state HLL approximate Riemann solver for ideal magnetohydrodynamics, J. Comp. Phys., 208, p. 315, (2005).

[16] E.F. Toro, M. Spruce, W. Speares, Restoration of the contact surface in the HLL Riemann solver, Shock Waves, 4, p. 25, (1994). 


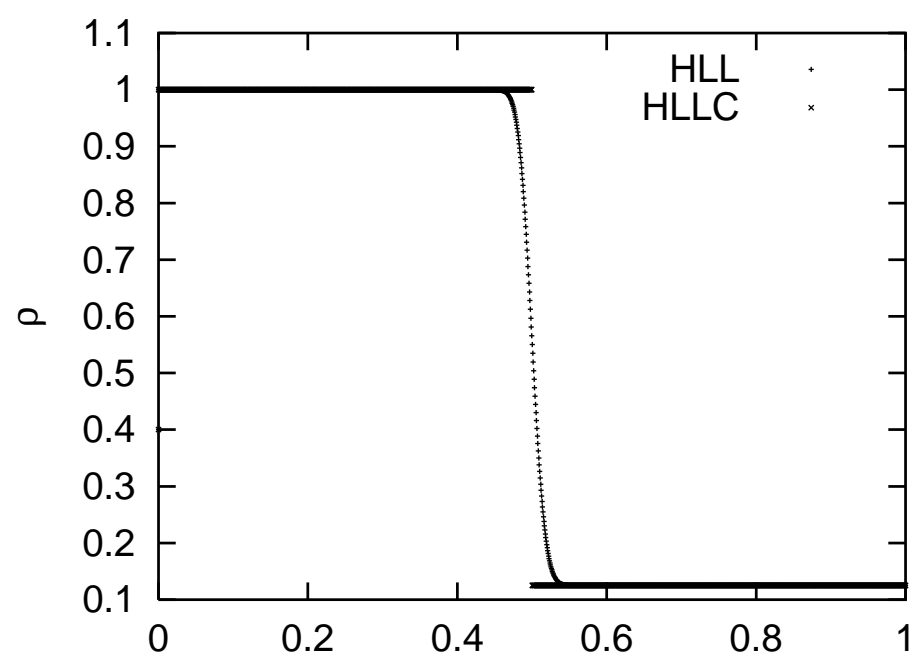

Figure 1: test 0a, HLL and HLLC

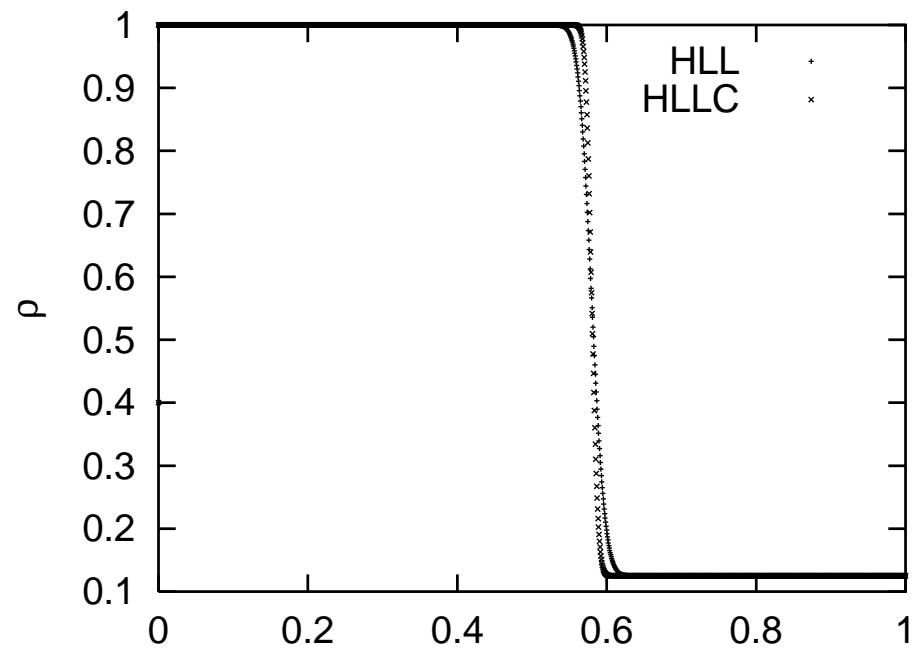

Figure 2: test 0b, HLL and HLLC 

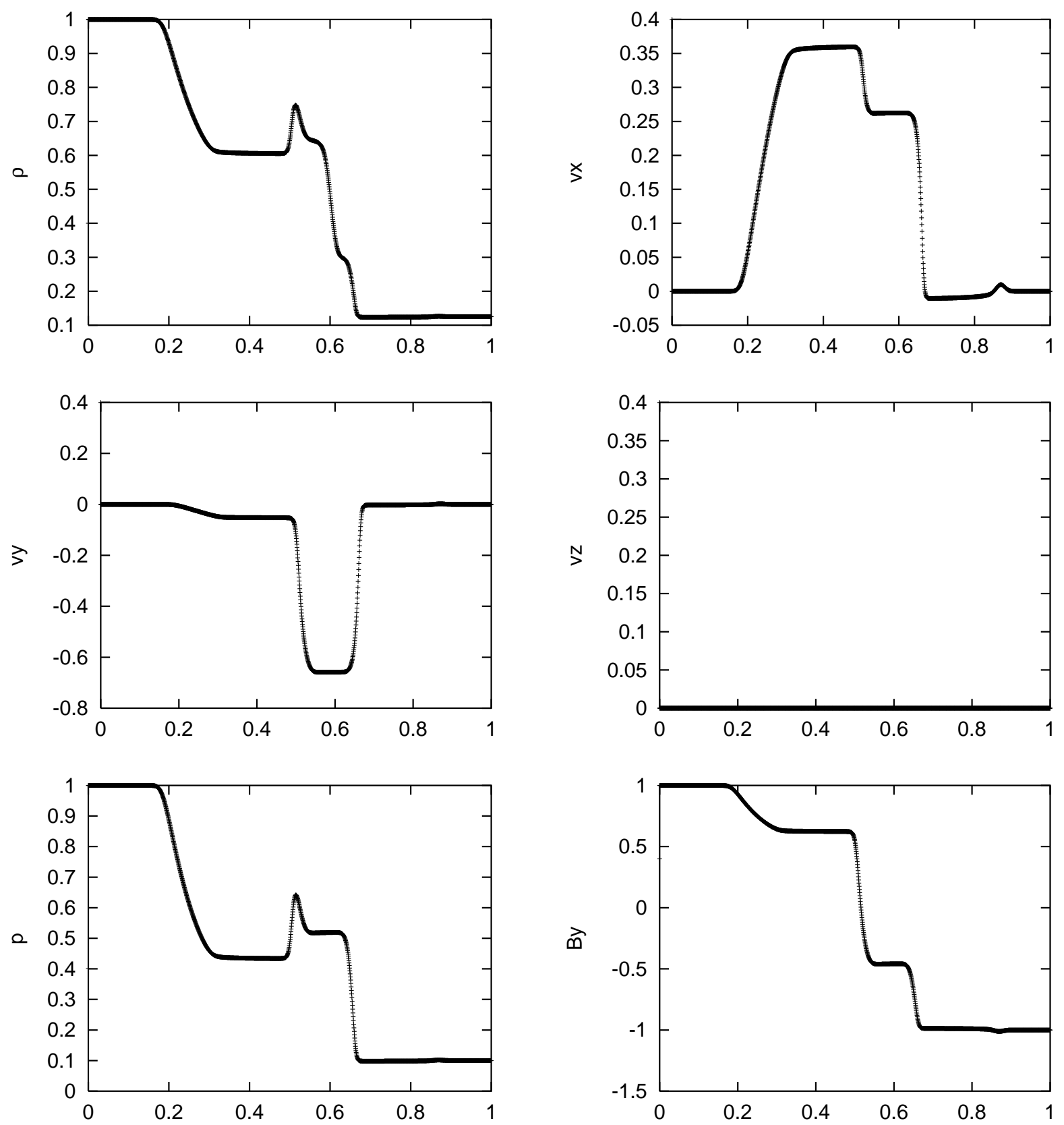

Figure 3: test 1, HLL 

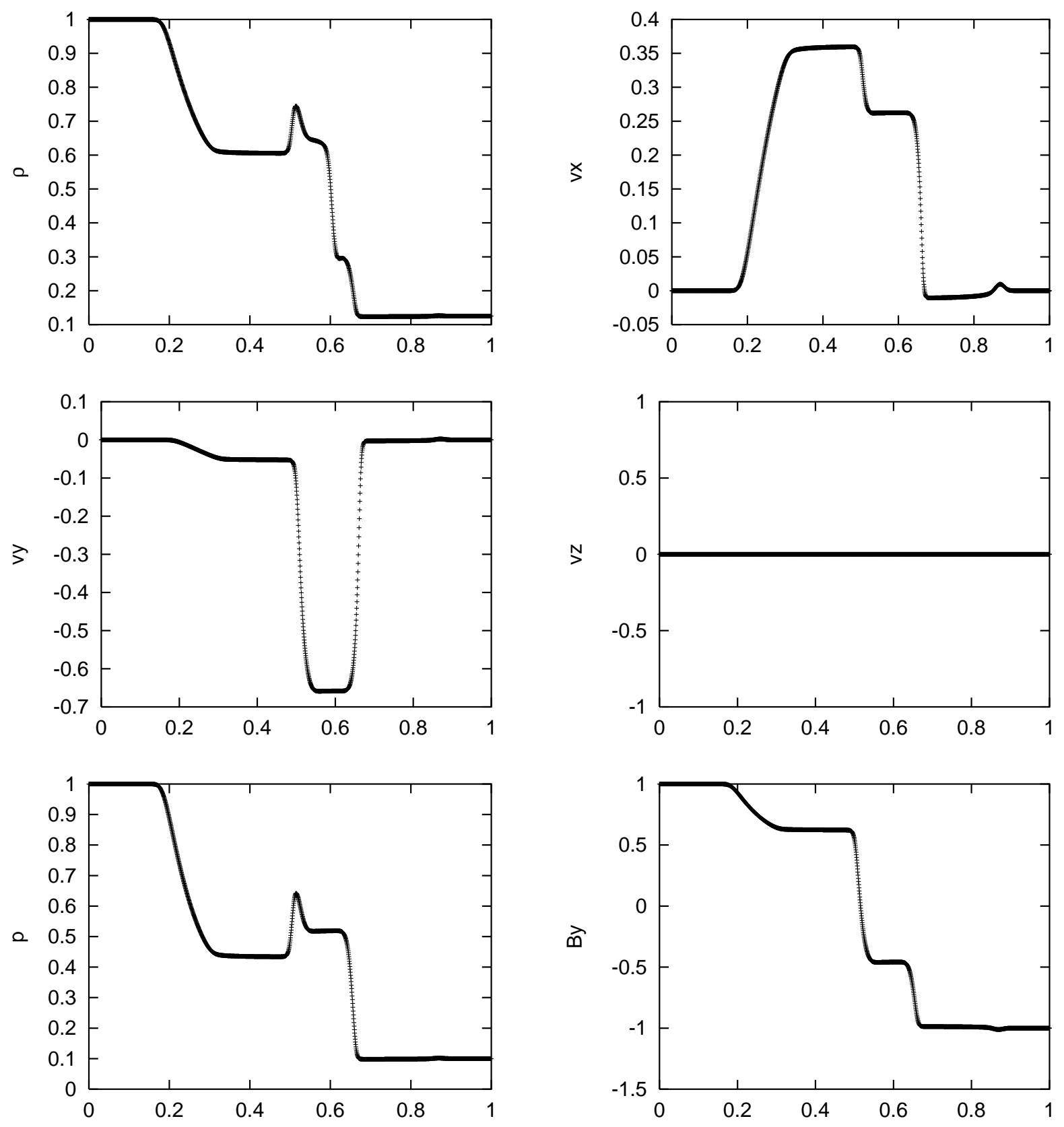

Figure 4: test 1, HLLC 

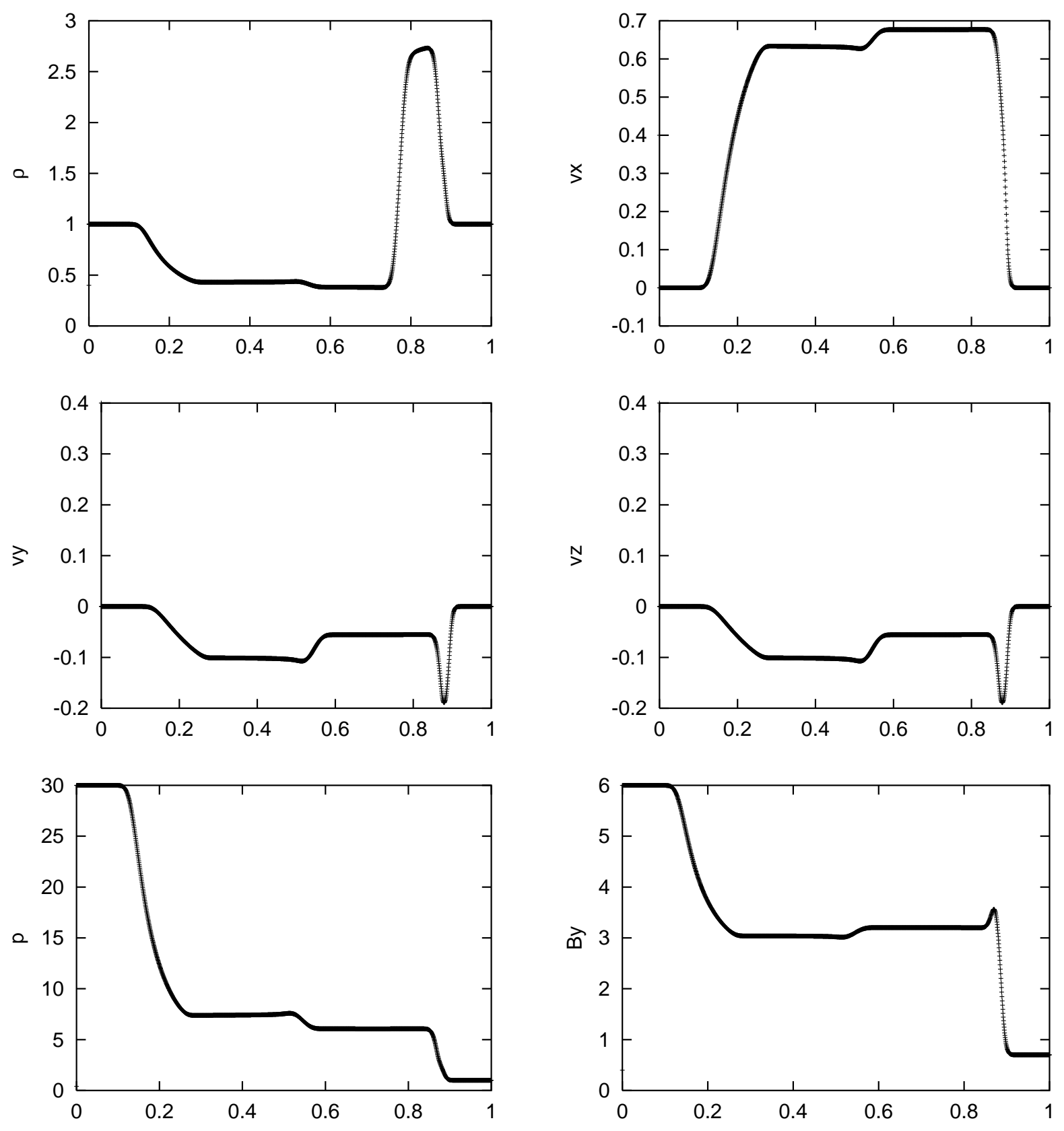

Figure 5: test 2, HLL 

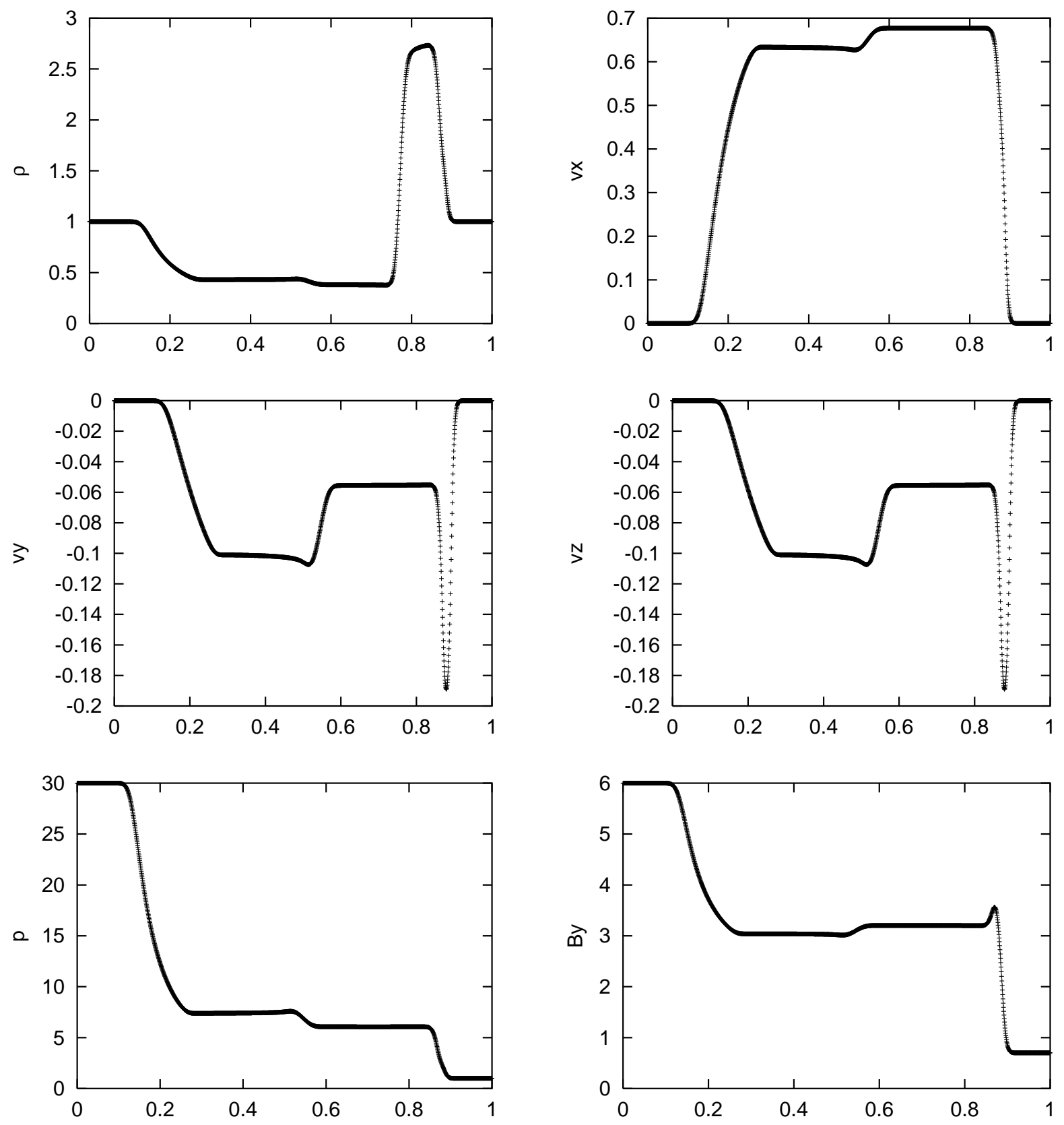

Figure 6: test 2, HLLC 

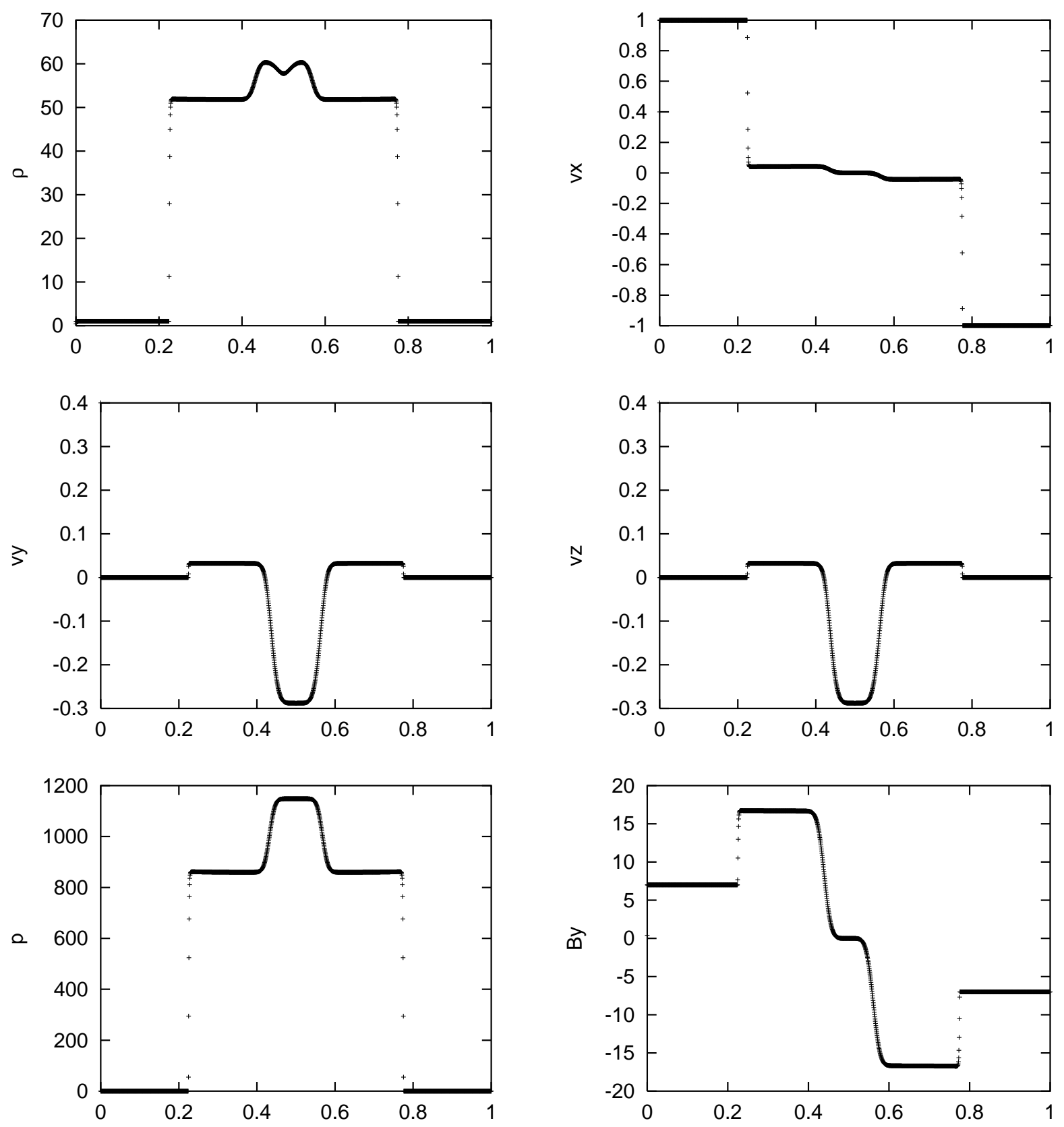

Figure 7: test 3, HLL 

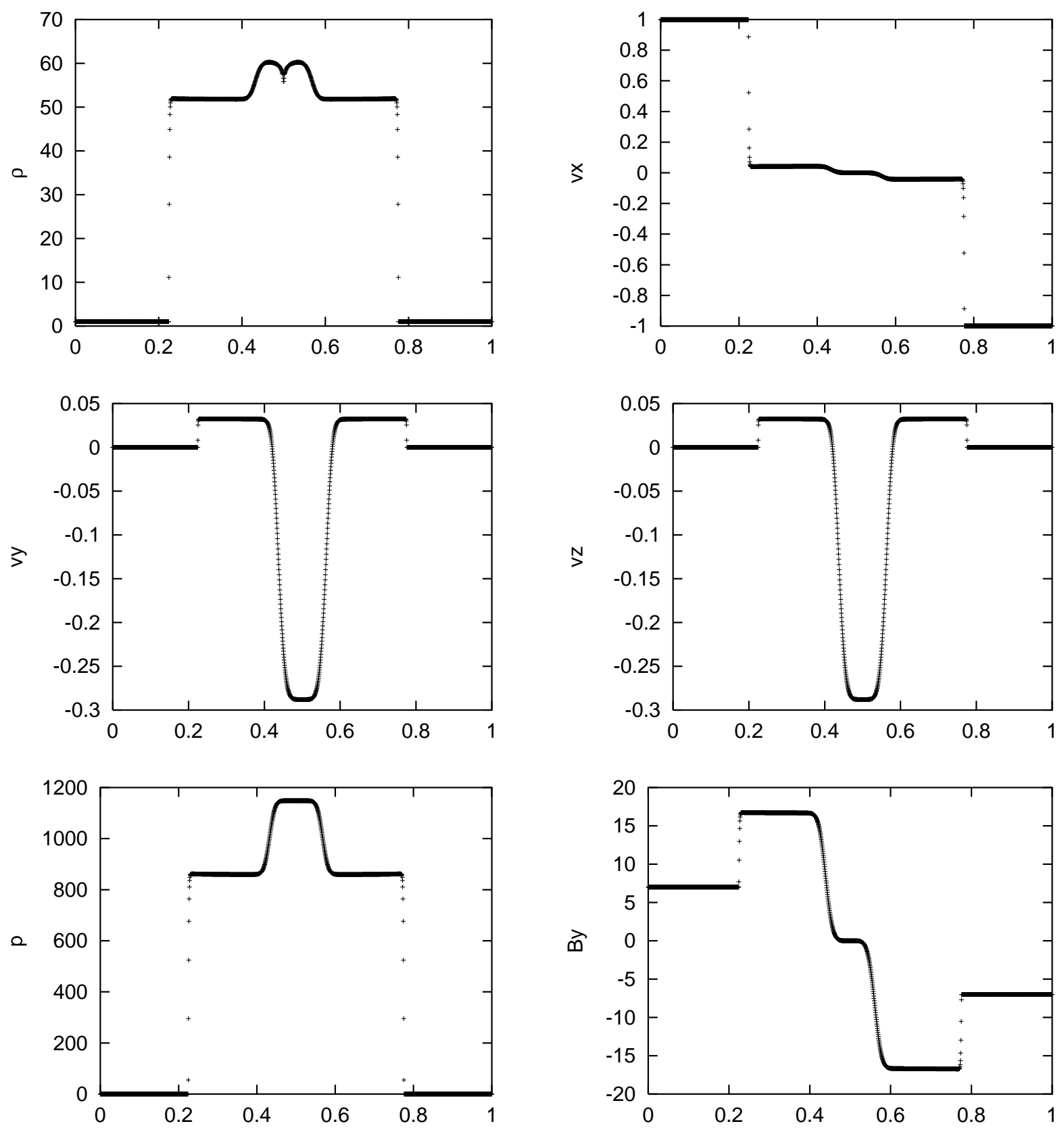

Figure 8: test 3, HLLC 

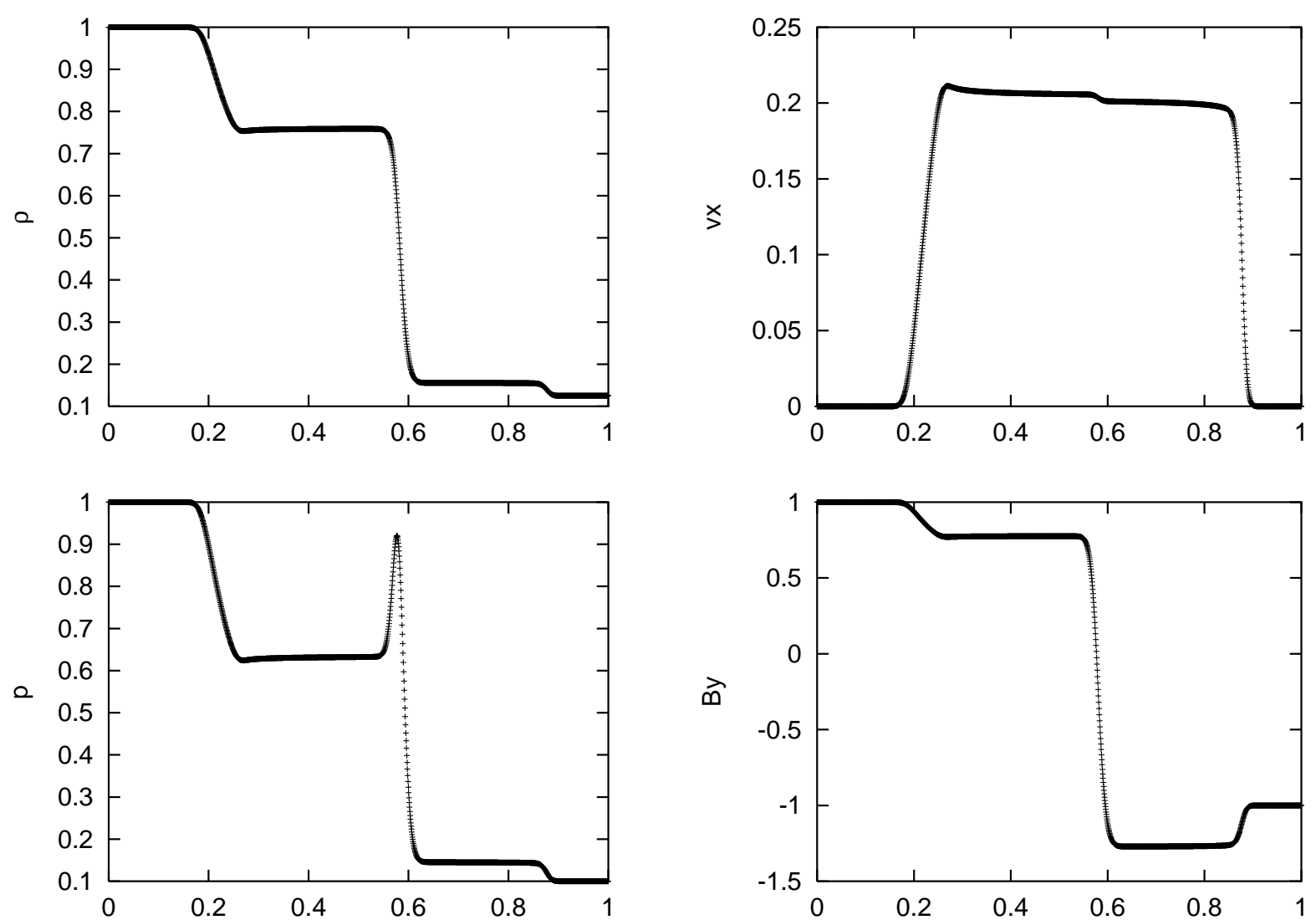

Figure 9: test 1b, HLL 

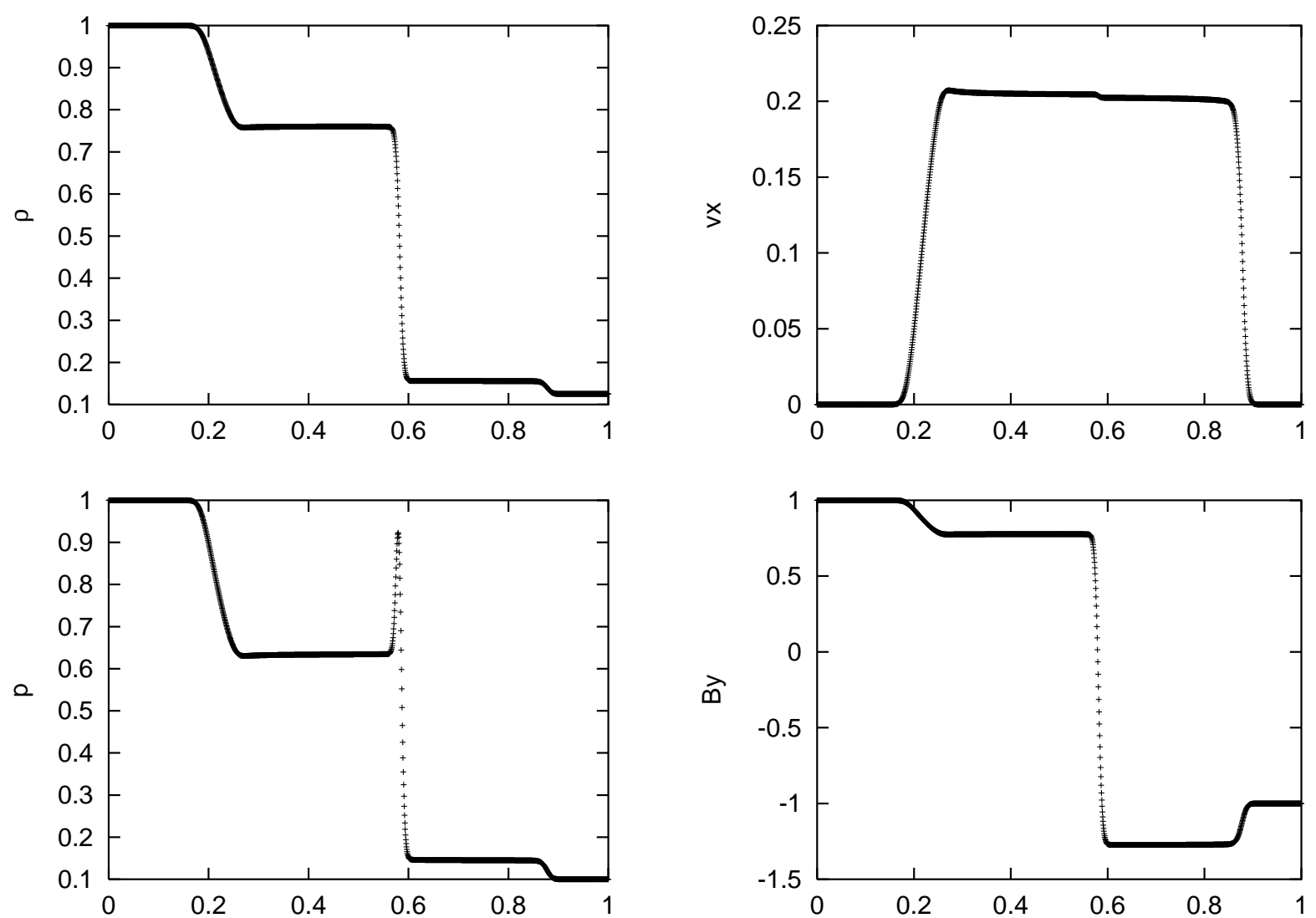

Figure 10: test 1b, HLLC 